\title{
Stochastic Modeling of Electrical Field in Potato Tuber us- ing Polynomial Chaos Expansion
}

\author{
Bartosz Sawicki ${ }^{1, *}$ and Artur Krupa ${ }^{1}$ \\ ${ }^{1}$ Warsaw University of Technology, Faculty of Electrical Engineering, pl. Politechniki 1, 00-950 War- \\ saw, Poland
}

\begin{abstract}
The paper deals with numerical modeling of objects with a natural origin. The stochastic approach based on description using random variables allows processing such challenges. The Monte-Carlo methods are known a tool for simulations containing stochastic parameters however, they require significant computational power to obtain stable results. Authors compare MonteCarlo with more advanced Polynomial Chaos Expansion (PCE) method. Both statistical tools have been applied for simulation of the electric field used in ohmic heating of potato tuber probes. Results indicate that PCE is remarkably faster, however, it simplifies some probabilistic features of the solution.
\end{abstract}

\section{Introduction}

The numerical modeling of objects of natural origin is a challenging topic. They are characterized by the complexity of internal structure and aleatoric variation of material parameters. Computational medicine is probably the most important scientific domain challenged by such variation of material properties [4,7]. But there are other applications in which numerical model has to deal with objects of natural origin, such as wood industry, mining of fossils or food processing. The last of them is subject of this paper.

Ohmic heating, also referred as direct resistance heating (DRH), is one of the well-known food processing techniques. Its main advantage is that the heat is provided in the same rate to a whole volume of a food sample. Numerical modeling is used to control and optimize the heating process [1]. In this paper, we model thermal power provided into the potato samples, but results could be easily expanded to the other plants.

The stochastic approach based on parameters described by random variables has been used to solve such problem. To analyze resistive heating phenomena in the plants one has to model power loses during current flow in the object. In case of standard deterministic materials it is considered as simple, but variable materials significantly complicate computations.

Methods for solving stochastic problems could be divided into two groups: intrusive which redefine whole mathematical description (e.g. Stochastic Finite Element Method [9]),

\footnotetext{
*e-mail: bartosz.sawicki@ee.pw.edu.pl
} 

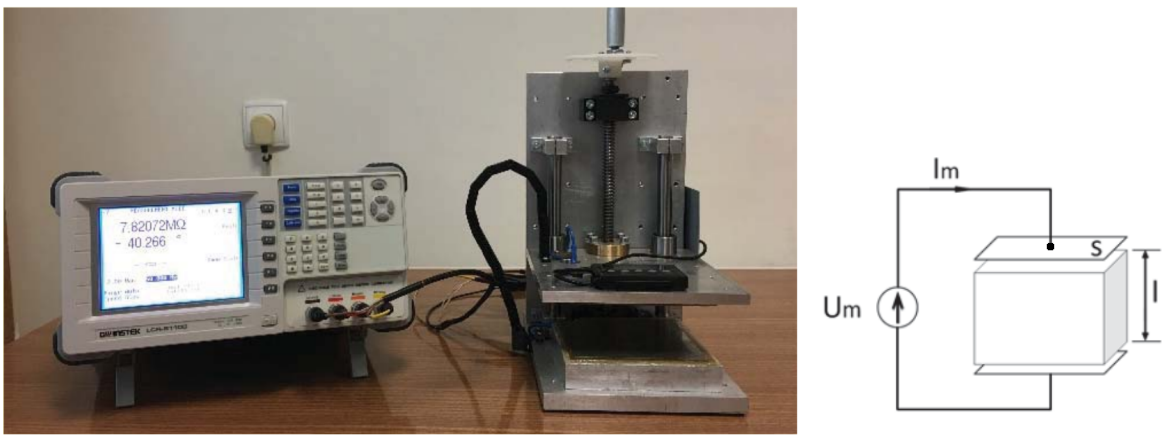

Figure 1: Picture and scheme of device constructed to measure electrical conductivity of solid materials with biological origin [6]

and non-intrusive where original deterministic solver is used as a part of the simulation algorithm. Intrusive methods are attractive and efficient for simple problems, nevertheless for large-scale simulations non-intrusive approach shows its benefits [5]. In the paper, two nonintrusive methods will be compared, Polynomial Chaos Expansion [8] with classical Monte Carlo sampling.

\section{Stochastic material parameters}

As it was mentioned already, materials with biological origin are significantly more complicated than technical ones. The small variability associated with manufacturing imperfections are replaced by a large natural variability of living organisms. For that reason, classical deterministic approach to material parameters has to be expanded to match the unpredictability of tissues. Stochastic methods based on random variables could be the solution to the problem.

However, the definition of probability density functions (PDF) requires a large number of independent samples. Literature review confirms significant variability of measurements [7], but it does not provide a systematic approach to the measurements.

Authors constructed a device to measure the electrical conductivity of solid biological samples [6]. As it is presented on Fig. 1, voltage $\left(U_{m}\right)$ and current $\left(I_{m}\right)$ are measured to get total resistance of the sample $\left(R_{m}=U_{m} / I_{m}\right)$. Device also provides values of geometrical dimensions of the measured cuboid ( $l$ - length, $s$ - contact surface). When contact resistivity $\rho_{c}$ is identified, electrical conductivity $\sigma$ could be found based on the formula:

$$
R_{m}=\frac{2 \rho_{c}}{s}+\frac{l}{\sigma s}
$$

Total measurement error for $\sigma$ has been identified as $10 \%$. It is defined mainly by the uncertainty of the contact resistance and also by imperfections of manual cuboid cutting procedure.

The main subject of the study is to model power dissipation in the potatoes when electrical resistive heating has been applied. Electrical conductivity of material is the essential value when power density is required.

Series of conductivity measurements have been performed for eight different potato varieties and also for other plants for comparison. Each of the variety has at least 20 independent 
a)
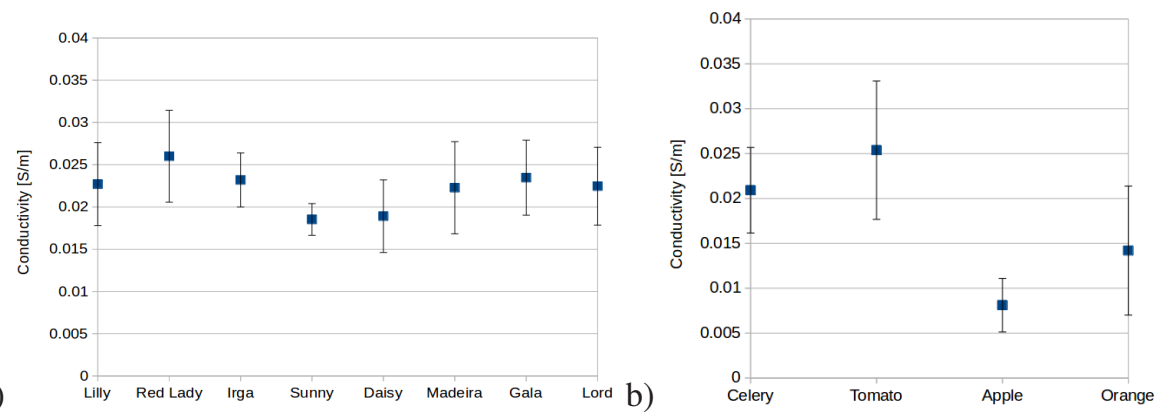

Figure 2: Conductivity measurements for 8 potato varieties (on the right) and 4 other plants (left).
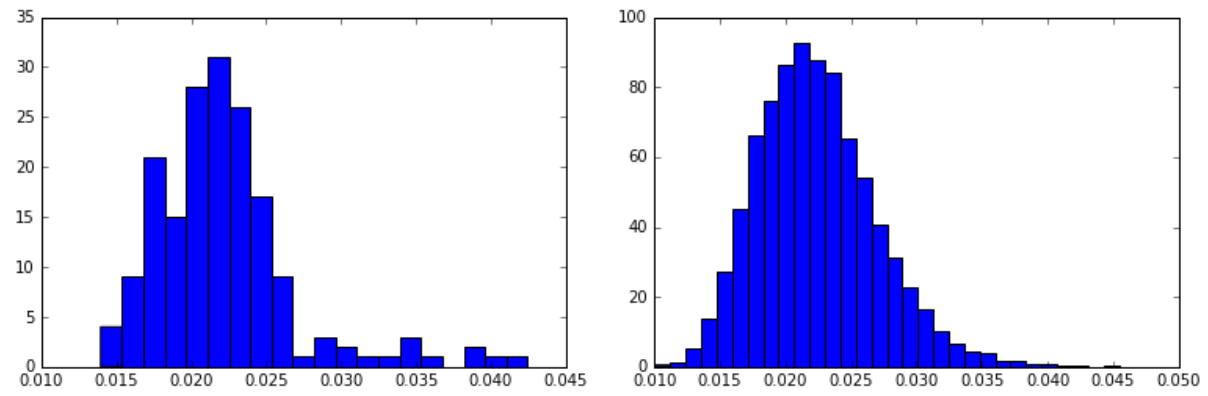

Figure 3: Histogram of 175 conductivity measurements of the raw potato tissue (right) and its lognormal PDF approximation (left).

measurements. Results of the measurements are presented as mean value and standard deviation on Fig. 2. As it is seen, the probes are characterized by high variability even in one variety. The variability level is about $20 \%$ for potato probes, here the highest value has been observed for 'Madeira' - 24\% and the lowest for 'Sunny' - 10\%. However potato tissue can be considered as a homogeneous, so variability is relatively low, comparing with other fruits and vegetables. For example, orange conductivity has a $50 \%$ spread.

Because we are interested in overall potato tissue property, all of the measured conductivities were combined in one single histogram (see Fig. 3). Then a shape of the conductivity probability has been identified as a log-normal distribution. It confirms previous expectation because this kind of shape is typical for variables describing naturally growing objects.

\section{Stochastic modeling}

Modeling of power density caused by current flow is described by Laplace equation and electric scalar potential $(\varphi)$ :

$$
\nabla \cdot \sigma \nabla \varphi=0
$$


with Dirichlet boundary condition forcing source voltage on the exciting electrodes. Then power density scalar field could calculated using formula:

$$
p=\vec{E} \cdot \vec{J}=\nabla \varphi \cdot \sigma \nabla \varphi
$$

where $\vec{E}$ is an electric field, and $\vec{J}$ is an current density field.

The model described above is a classic solution with deterministic parameters, and is solved by the Finite Element Method. Thus we created model $f$ which is a function of input parameters describing the geometry of the object, material parameter $(\sigma)$ and sources.

Stochastic modeling is an extension in which it is assumed that input is the subject of unpredictable variation. In our model we treat all of the input parameters as described by the random variables $(Q)$ :

- conductivity distribution (random field),

- geometrical dimensions of the sample (cuboid),

- value of the source voltage.

The reasoning for random conductivity distribution has been discussed in the previous section with a conclusion about log-normal PDF for $\sigma$. The other parameters such as the geometrical size of the sample and value of the source voltage are also subject of uncertainty, but with normal Gaussian distribution. Variability of the dimensions is set to be $10 \%$. The voltage is more precisely controlled, so $1 \%$ spread has been assumed.

To solve such a stochastic problem we decided to use non-intrusive methods. The main reason for that is that they don't introduce a need to modify forward simulation software, what has two important advantages. First of all, finite element simulation code can be kept unchanged and optimized. The second advantage, which may be even more important, is the fact that the methods are easy to run in the parallel computational environment.

In this paper two non-intrusive methods will be compared directly:

Monte Carlo is a method based on sampling solution space in a random way. Random generators prepare sets of input parameters $(Q)$, then model is solved in classical, deterministic way using forward model $f(Q)$. Monte Carlo is simple method to implement, it also preserves complete statistic information. On the other hand it is known as the one with very low convergence. It requires large computational resources to find stable solution.

Polynomial Chaos Expansion (PCE) is a method based on construction of approximation model $\hat{f}(Q)$ by polynomial expansion with order $m$ :

$$
\hat{f}(Q)=\sum_{i=0}^{m} c_{i} \Phi_{i}(Q)
$$

where $c_{i}$ are coefficients, and $\Phi_{i}$ orthogonal polynomials. Statistical properties of $f(Q)$ could be analyzed through low cost numerical computations where $\hat{f}(Q)$ is used as a surrogate for $f(Q)$.

\section{Simulation results}

In the numerical experiment potato sample with dimensions $3 \times 4 \mathrm{~cm}(10 \%$ standard deviation) has been modeled. The probe is connected to the voltage source $(30 \mathrm{~V})$ by using two 


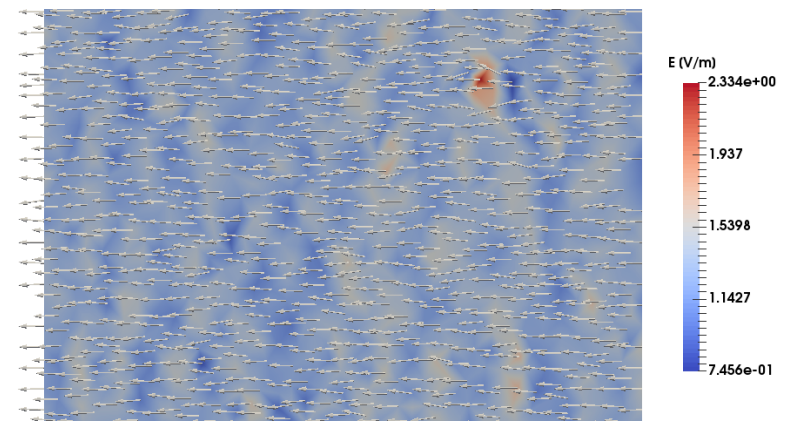

Figure 4: Result of single forward simulation of electric field inside a potato with random conductivity distribution

electrodes which are placed on opposite sides. Because of the simple geometry model is considered as a two-dimensional. Distribution of conductivity parameter of potato is described by a random field with log-normal probability density function determined in the previous section (see Fig. 3) and grain size is set to be $1 \mathrm{~mm}$.

Forward field distribution is calculated according to the eq. 2 by the Finite Element Method solver that has been developed with the FEniCS library. FEniCS is a specialized and efficient open-source software with Python language interface [2]. The single forward simulation takes about 3-5 seconds. Example of electric field distribution is shown in Fig. 4. One can notice how random variation of conductivity disturbs propagation of the homogeneous source oriented horizontally.

We are interested in statistical properties of the power dissipated in the potato sample, so calculated electric field is transformed into power density (according to eq. 3) and then integrated over the whole volume to obtain total power.

Stochastic simulations have been done using Chaospy software [3]. It is a Python software toolbox for performing uncertainty quantification. Chaospy has a large number of features supporting analysis with Monte Carlo and PCE methods.

The first experiment is related to the Monte Carlo method. Results presented on Fig. 5 confirmed that the method has a low convergence. After 10 or even 30 forward simulations its hard to recognize the shape of probability density function of the solution. In our case, about 200 iterations (about 1000 seconds) were required to realize normal-gaussian distribution of dissipated power. On the other hand, the method is easy to implement in parallel architectures and does not introduce any simplifications, what are important advantages. Statistical measures of probability distributions are presented in Table 1 . The solution with 1000 iterations could be treated as a reference with proper values of skewness and kurtosis, however, simulation took nearly 3000 seconds.

In the second part of the study polynomial expansion of the model has been calculated according to eq. 4. Coefficients of orthogonal polynomials have been built using regression of the small number of randomly generated samples and their evaluations. The main advantage of the method is that a low number of Monte Carlo samples is enough for define polynomial expansion of the model. The number of required samples and order of polynomial should be matched to the problem. 


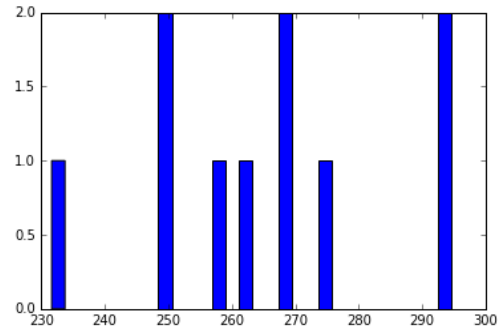

(a) $\mathrm{n}=10$

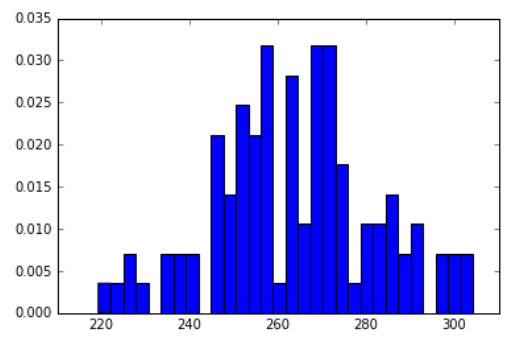

(c) $\mathrm{n}=100$

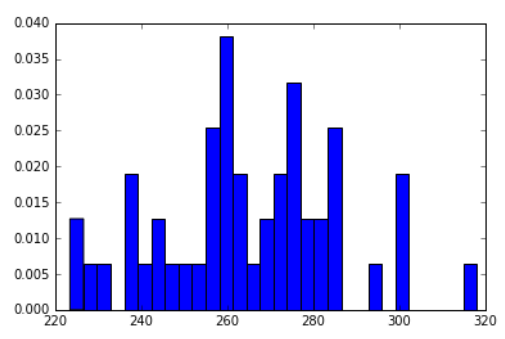

(b) $n=30$

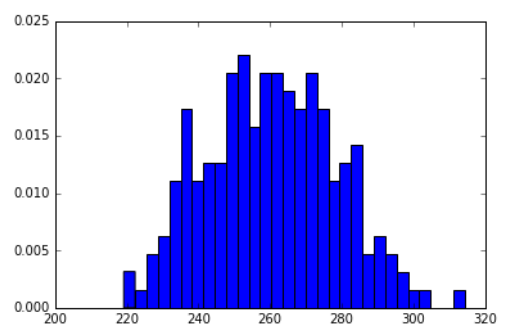

(d) $n=200$

Figure 5: Histogram for Monte Carlo simulations with different number of samples

Table 1: Statistical measures of results with simulation time

\begin{tabular}{|l|c|c|c|c||c|}
\hline & mean & std. dev. & skewness & kurtosis & time $[\mathrm{s}]$ \\
\hline \hline Monte Carlo, $\mathrm{n}=10$ & 265.42 & 14.72 & 0.333 & -0.268 & 30 \\
\hline Monte Carlo, $\mathrm{n}=30$ & 265.96 & 18.62 & -0.363 & -0.695 & 93 \\
\hline Monte Carlo, $\mathrm{n}=100$ & 264.17 & 20.01 & 0.11 & 0.303 & 290 \\
\hline Monte Carlo, $\mathrm{n}=1000$ & 264.50 & 19.40 & 0.238 & 0.242 & 2943 \\
\hline \hline PCE, $\mathrm{n}=10, \mathrm{~m}=0$ & 269.44 & 0 & 0 & -3 & 31 \\
\hline PCE, $\mathrm{n}=10, \mathrm{~m}=1$ & 263.06 & 20.38 & 0.002 & -0.022 & 31 \\
\hline PCE, $\mathrm{n}=10, \mathrm{~m}=2$ & 196.64 & 266.87 & -1.024 & 1.559 & 31 \\
\hline PCE, $\mathrm{n}=10, \mathrm{~m}=3$ & 331.89 & 231.33 & 3.507 & 26.15 & 31 \\
\hline
\end{tabular}

As seen on the lower part of Table 1 simulation time are always the same (31 seconds). The computational complexity of polynomial expansion is nearly negligible, comparing to the complexity of finite element electromagnetic field simulations, which for $n=1$ took about 30 seconds. Only first order polynomials $(\mathrm{m}=1)$ give correct results. For other cases, statistical measures of the result are strongly damaged. The best PCE result with order 1 is visually compared with wrong results for order 3 on Fig. 6.

Our experiments have shown that the PCE method is very fast, however, it has a negative impact on the result probability distribution. Especially higher order statistical measures (skewness and kurtosis) are affected. 
a)

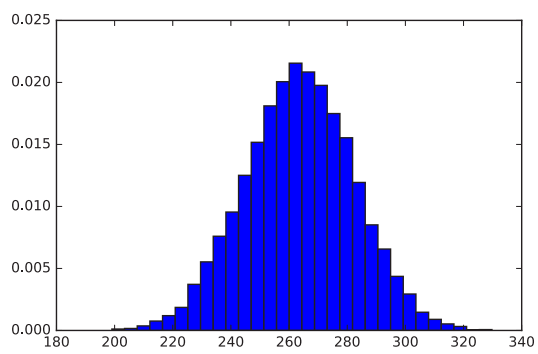

Figure 6: Results of PCE reconstructed distribution, a) $n=10, m=1$; b) $n=10, m=3$

\section{Conclusions}

Developed model of power dissipation in the potato tuber allowed to perform stochastic simulation, and identify probability density function of the power. It has been confirmed that Polynomial Chaos Expansion method is computationally cheap, however, it introduces simplifications which interfere with higher order statistical measures. Monte Carlo does not have such drawbacks. Proper choice of the method should be based on the accepted tolerance of probability distribution.

Further works will be focused on the modeling of more inhomogeneous materials, which are a mixture of different components. In such cases, time-consuming Monte Carlo method will be the first choice, so special efforts should be placed on the parallelization of the simulation platform.

\section{References}

[1] A.A. De Alwis, P.J. Fryer: A finite element analysis of heat generation and transfer during ohmic heating of food. Chemical Engineering Science, vol. 45, pp. 1547-1559, 1990

[2] M. S. Alnæs, J. Blechta, J. Hake, et. al., The FEniCS Project Version 1.5, Archive of Numerical Software, vol. 3, No. 100, pp. 9-23, 2015.

[3] Jonathan Feinberg, Hans Petter Langtangen. Chaospy: An open source tool for designing methods of uncertainty quantification, Journal of Computational Science, vol. 11, pp. 4657,2015

[4] Paul Hauseux, Jack Hale, S. Cotin, Stéphane Bordas: Accelerating Monte Carlo estimation with derivatives of high-level finite element models, Computer Methods in Applied Mechanics and Engineering, 2017

[5] Paul Hauseux, Jack Hale, S. Cotin, Stéphane Bordas: Quantifying the uncertainty in a hyperelastic soft tissue model with stochastic parameters, Preprint submitted to Applied Mathematical Modelling February 9, 2018

[6] Artur Krupa, Bartosz Sawicki: Measurement-based stochastic models of biological materials, 2017 18th International Conference on Computational Problems of Electrical Engineering (CPEE), Kutna Hora, 2017, pp. 1-4, doi: 10.1109/CPEE.2017.8093068

[7] Bartosz Sawicki: Uncertainty of numerical simulations in bioelectromagnetic problems, Przeglad Elektrotechniczny 91, pp. 50-54 (2015) 
[8] Yang Shuxing, Fenfen Xiong, and Fenggang Wang: Polynomial Chaos Expansion for Probabilistic Uncertainty Propagation Uncertainty Quantification and Model Calibration. InTech, 2017.

[9] George Stefanou: The stochastic finite element method, Past, present and future, Computational Methods Applied in Mechanical Engineering, vol. 19, pp. 1031-1051, 2009 